\title{
A first missense mutation in the $\delta$ sarcoglycan gene associated with a severe phenotype and frequency of limb-girdle muscular dystrophy type 2F (LGMD2F) in Brazilian sarcoglycanopathies
}

\author{
Eloisa S Moreira, Mariz Vainzof, Suely K Marie, Vincenzo Nigro, Mayana Zatz, \\ Maria Rita Passos-Bueno
}

\section{Departamento de Biologia, Instituto de Biociências, Universidade de São Paulo, Rua do Matão 277, Sala 211, CEP 05508-900, São Paulo, SP, Brazil E S Moreira $M$ Vainzof M Zatz \\ M R Passos-Bueno}

Departamento de Neurologia, Faculdade de Medicina, Universidade de São Paulo, Avenida Dr Arnaldo 455, Sala 4110, São Paulo, SP, Brazil $M$ Vainzof

S K Marie

Instituto di Patologia Generale e Oncologia, Facoltà di Medicina, Seconda Università degli studi di Napoli, Larghetto S Aniello a Caponapoli, 2-80138, Napoli, Italy

V Nigro

Correspondence to:

Dr Passos-Bueno.

Received 10 December 1997 Revised version accepted for publication 3 April 1998

\begin{abstract}
Among the heterogeneous group of autosomal recessive limb-girdle muscular dystrophies (AR LGMDs), the sarcoglycanopathies (LGMD2C-2F) represent a subgroup characterised by defects in the $\gamma, \alpha, \beta$, and $\delta$ sarcoglycan genes, respectively. Genotype-phenotype correlations in these forms of AR LGMD are important to enhance our understanding of protein function. Regarding LGMD2F, only two homozygous frameshift mutations have been reported to date in patients with a severe phenotype. In the present report, through screening 23 unrelated AR LGMD patients, we identified three subjects with LGMD2F, two with a previously reported frameshift mutation and the other homozygous for a new missense mutation in the $\delta$ sarcoglycan gene. Interestingly, this new mutation is also associated with a severe clinical course. In addition, our results suggest that this form of severe AR LGMD is not very rare in our population.

(F Med Genet 1998;35:951-953)
\end{abstract}

Keywords: sarcoglycanopathies; limb-girdle muscular dystrophies; $\delta$ sarcoglycan; linkage disequilibrium

The sarcoglycanopathies, a subgroup of autosomal recessive limb-girdle muscular dystrophies (AR LGMDs), are characterised by progressive muscle weakness affecting both upper and lower limbs. ${ }^{12}$ In the last few years, four sarcoglycanopathies, named LGMD2C, ${ }^{3}$ LGMD2D, ${ }^{4}$ LGMD2E, ${ }^{56}$ and LGMD2F, have been identified. They were found to be caused by mutations in the $\gamma(13 \mathrm{q} 12),{ }^{8} \alpha$ $(17 \mathrm{q} 21.1),{ }^{49^{10}} \beta(4 \mathrm{q} 12),{ }^{56}$ and $\delta(5 \mathrm{q} 33)^{11}{ }^{12}$ sarcoglycan genes, respectively. The sarcoglycans are transmembrane glycoproteins which, together with dystrophin, dystroglycans, and syntrophins, form the "dystrophinglycoprotein complex" (DGC). ${ }^{13-15}$

A vast repertory of missense, nonsense, and frameshift mutations have been found in the $\alpha$, $\beta$, and $\gamma$ sarcoglycan genes in patients with AR LGMD, ${ }^{4-616-22}$ but linkage disequilibrium was only found for the $\Delta 521-\mathrm{T}$ mutation in the $\gamma$ sarcoglycan gene. ${ }^{20}{ }^{23}$ Until now, it has not been possible to establish a clear genotypephenotype correlation.
The LGMD2F form was first reported in four Brazilian families, where all the affected patients share a homozygous frameshift mutation in exon 7 of the $\delta$ sarcoglycan gene (del656C), resulting in the premature truncation of the translatable protein. ${ }^{12}$ This mutation is apparently in linkage disequilibrium with alleles of two microsatellite markers of the 5q33-34 region. ${ }^{12}$ Recently, Duggan et $a l^{4}$ described a girl with a homozygous nonsense mutation in codon 165 (R165X) of the $\delta$ sarcoglycan gene. ${ }^{24}$ All the LGMD2F patients reported so far show a DLMD phenotype, with complete absence of the whole sarcoglycan complex in muscle. ${ }^{12425}$

The aims of the present report are: (1) to describe the haplotype associated with the del656C mutation in Brazilian families; (2) to identify other mutations in Brazilian patients affected by this form of sarcoglycanopathy; and (3) to estimate the frequency of the LGMD2F form among our DLMD families.

Twenty-five unrelated DLMD families were included in the present study: two large families for linkage analysis (LG26 and LG43) and 23 small pedigrees for identification of LGMD2F patients. Clinical data on patients from families LG26, LG33, LG43, and LG68 are detailed elsewhere. ${ }^{72}$ Among the 21 new cases, it was possible to perform muscle biopsy in 10 , who showed a negative staining pattern for $\alpha$ sarcoglycan.

A total of 15 microsatellite markers of the 5q33-34 region were tested in the two families in which we first detected linkage (LG26 and LG43). ${ }^{7}$ Among them, we observed that the affected patients from both families share the same homozygous haplotype for markers D5S487 and D5S412. The frequencies of the alleles from these two markers that are segregating with the disease allele were estimated in normal chromosomes. Allele 1 (188 bp) of D5S412 was present in only one in 80 chromosomes in the healthy population. Interestingly, allele 10 (236 bp) of D5S487 was not found in 204 normal chromosomes from our population, including whites and AfricanNegroids. Interestingly, this allele, which is apparently being reported here for the first time, is $17 \mathrm{bp}$ smaller than the smallest allele (allele $8=253 \mathrm{bp}$ ) published so far. In order to exclude the possibility that our control sample was not representative, we have genotyped it for marker D13S232, since the 122 bp allele from this locus is in linkage disequilibrium with 


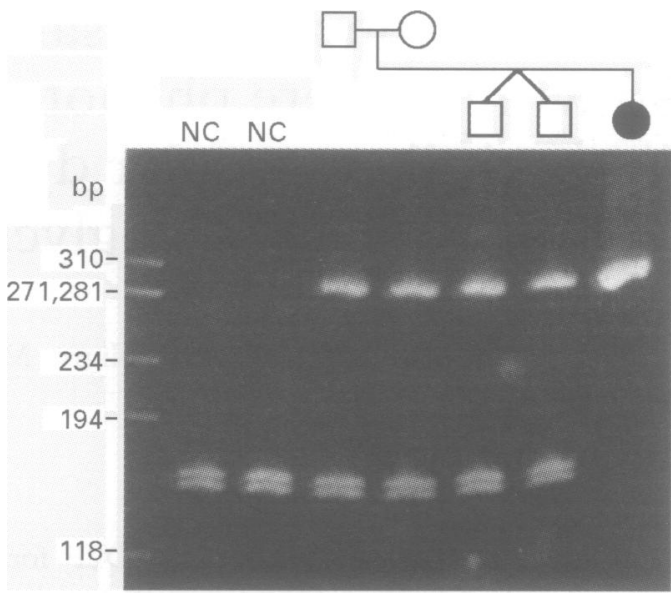

Figure 1 TaqI digestion of the exon 8 PCR products of the $\delta$ sarcoglycan gene, showing that the affected patient has lost the restriction site for this enzyme because of the E262K mutation. The parents and her normal sibs are carriers of this mutation. NC=normal control.

LGMD2C. ${ }^{24}$ Among 144 tested chromosomes, three had the $122 \mathrm{bp}$ allele. The obtained frequency of 0.021 is consistent with that found in white controls $(0.023),{ }^{24}$ suggesting therefore that our control sample is representative of our population.

Therefore, among the 15 markers tested that are segregating with the disease gene, it was found that allele 1 of D5S412 is rare, while allele 10 of D5S487 is very rare in the control population, supporting evidence of a strong linkage disequilibrium between each of these alleles and the pathogenic mutation del656C. Based on these findings, we tested D5S412 and D5S487 in 23 small DLMD families (with one or two affected sibs). We found only the two previously reported unrelated patients, from kindreds LG33 and LG68, ${ }^{12}$ to be homozygous for alleles 1 and 10 of markers D5S412 and D5S487, respectively. As previously shown, these patients have the same homozygous missense mutation (del656C) found in the LG26 and LG43 affected subjects, ${ }^{12}$ confirming the strong linkage disequilibrium between this mutation and the haplotype $1 / 10-1 / 10$ (D5S412/D5S487).

Single strand conformation polymorphism (SSCP) analysis for exons 2 to 8 of the $\delta$ sarcoglycan gene was performed in the remaining 21 DLMD patients. One affected girl showed a different migration pattern for exon 8 compared to the normal population. The sequencing of this exon detected a $\mathrm{C} \rightarrow \mathrm{A}$ transversion at position 784 of the cDNA, which causes the substitution of a glutamic acid for a lysine at residue $262(\mathrm{E} 262 \mathrm{~K})$ of the translatable protein. This mutation was confirmed after digestion of the PCR product with TaqI, since it abolishes a restriction site for this endonuclease. In addition, the parents and the normal sibs of this patient were found to be heterozygous for the E262K mutation (fig 1). This affected girl of normal intelligence, currently 15 years old, belongs to a consanguineous white kindred (LG71) and has three normal sibs. Her parents report that she had a normal neuropsychomotor development. At the age of 8 , she had difficulty in walking and climbing stairs, followed by pain and cramps.
At the age of 11, when first seen by us, she had hypertrophy of the calves and a Gowers sign. She was confined to a wheelchair at 12 years and at present she is unable to raise her arms thus showing a severe DLMD course. A recent electrocardiogram (ECG) showed hypertrophyD and involvement of the left ventricle. Muscle biopsy showed a dystrophic pattern, with positive immunofluorescence staining for dys trophin, a total absence of $\delta$ sarcoglycan, accompanied by a total absence of $\alpha$ and $\beta$ sar- $\frac{\bar{c}}{\bar{\omega}}$ coglycans, but only a partial deficiency of $\gamma_{\bar{\Phi}}$ sarcoglycan. ${ }^{26}$

To our knowledge, the substitution of onen amino acid, in the present case the glutamica acid in position 262 , is the first missense muta- $-\overrightarrow{-}$ tion found in the $\delta$ sarcoglycan gene. Interest $-\vec{\omega}$ ingly, it causes a phenotype as severe as that presented by the other LGMD2F patients witho truncating mutations. ${ }^{1224}$ This glutamic acider residue, which is localised in the $\delta$ sarcoglycan extracellular domain two residues upstream ofe the cysteine cluster, is very conserved among different species and it is also present in the corresponding region of the $\gamma$ sarcoglycan. $\vec{z}$ This cysteine rich region, which is a conservedo region among all the sarcoglycan family members, seems to participate in intra- ando intermolecular disulphide bond formation. ${ }^{1 \stackrel{\Phi}{-}}$ Therefore, the mutation described here occursec in an important domain of the $\delta$ sarcoglycan,,$\infty$ interfering with the conformation of the tertiary structure of this molecule. Theses observations, together with the fact that this change was not found among 100 chromosomes from the healthy population, strongly@ suggest that the $\mathrm{E} 262 \mathrm{~K}$ mutation is patho- $\overrightarrow{\hat{\sigma}}$ genic. This mutation is associated with a3 haplotype distinct from the one found in the? other LGMD2F patients, in which the del656C mutation was identified. Interestingly, all the families with del656C have an African ancestry, in contrast to the family with the E $262 \mathrm{~K}$ mutation, who is white. Therefore these data suggest that the del656C might have 3 originated in Africa and spread in the Brazilian population or alternatively that this haplotype predisposes to the occurrence of this mutation. In this respect, it will be important to verify if the del656C is also found in association witho different haplotypes.

Considering the 23 small DLMD Brazilian N families screened for mutations in the $\delta 0$ sarcoglycan gene, we estimated that the frequency of cases resulting from mutations in this locus is $13 \%(3 / 23)$ in our population. If we? take into account only the 11 families in which궁 $\alpha$ sarcoglycan deficiency could be confirmed, $\stackrel{\vec{\Phi}}{\mathbb{D}}$ this frequency is even greater, in accordance? with the proportion of nearly $30 \%$ of $\delta \stackrel{\mathbb{Q}}{ }$ sarcoglycan mutations among the four sar? coglycanopathies recently estimated in a large Brazilian sample. ${ }^{27}{ }^{28}$ Therefore, the LGMD2FO form is apparently not rare among our DLMD흘. families, as compared to the data recently? reported by Duggan et $a l,^{24}$ in which a frequency of $\sim 4 \%(2 / 54)$ was estimated for LGMD2F among families with $\alpha$ sarcoglycan deficiency. 
It will be interesting to verify if the mutation E262K is present in other patients, particularly of white ancestry, with LGMD2F. This will be important to elucidate the origin of mutations in this gene as well as to improve our understanding of the function of $\delta$ sarcoglycan.

We are extremely grateful to Constancia G Urbani, Flávia D Albuquerque, Antonia M P Cerqueira, Marta Canovas, Simone Albuquerque, Antonia M P Cerqueira, Marta Canovas, Simone
Campiotto, Dr Rita C M Pavanello, Dr Ivo Pavanello Filho, Campiotto, Dr Rita C M Pavanello, Dr Ivo Pavanello Filho, Andréa L Sertié, Cleber S Costa, and all the patients and their
relatives. This work was supported by grants from the Fundação de Amparo à Pesquisa do Estado de São Paulo (FAPESP), the Programa de Apoio ao Desenvolvimento Científico e Tecnológico (PADCT), the Financiadora de Estudos e Projetos (FINEP), the Conselho Nacional de Desenvolvimento Cientifico e Tecnológico (CNPq), the Associação Brasileira de Distrofia Muscular (ABDIM), the International Agency of Atomic Energy (IAAE), and the PRONEX. The research of MRPB was supported in part by an International Research MRPB was supported in part by an International Resea
Scholars grant from the Howard Hughes Medical Institute.

1 Walton JN, Gardner-Medwin D. The muscular dystrophies. In: Walton JN, ed. Disorders of voluntary muscle. 5 th ed. New York: Churchill Livingstone, 1988:519-68.

2 Bushby K, Beckmann J. Report of the 30th and 31st ENMC International Workshop: the limb-girdle muscular dystrophies and proposal for a new nomenclature. Neuromusc Disord 1995;5:337-43.

3 Othmane $K B$, Ben Hamida $M$, Pericak-Vance MA, et al. Linkage of Tunisian autosomal recessive Duchenne-like muscular dystrophy to the pericentromeric region of chromuscular dystrophy to the pericentromeric

4 Roberds SL, Leturcq F, Allamand V, et al. Missense mutations in the adhalin gene linked to autosomal recessive muscular dystrophy. Cell 1994;78:625-33.

5 Bönnemann CG, Modi R, Noguchi S, et al. $\beta$-sarcoglycan (A3b) mutations cause autosomal recessive limb-girdle muscular dystrophy with loss of the sarcoglycan complex. Nat Genet 1995;11:266-73.

6 Lim LE, Duclos F, Broux O, et al. $\beta$-sarcoglycan: characterisation and role in limb-girdle muscular dystrophy linked to 4q12. Nat Genet 1995;11:257-65.

7 Passos-Bueno MR, Moreira ES, Vainzof M, et al. Linkage analysis in autosomal recessive limb-girdle muscula dystrophy (AR LGMD) maps a sixth form and indicates that there is at least one more subtype of AR LGMD. Hum Mol Genet 1996;5:815-20.

8 Noguchi S, McNally EM, Othmane KB, et al. Mutations in the dystrophin-associated protein $\gamma$-sarcoglycan in chromosome 13 muscular dystrophy. Science 1995;270:819-22.

9 Roberds SL, Anderson RD, Ibraghimov-Beskrovnaya O, et al. Primary structure and muscle-specific expression of the $50-\mathrm{kDa}$ dystrophin-associated glycoprotein (adhalin). $\mathcal{f}$ Biol Chem 1993;268:23739-42.

10 McNally EM, Yoshida M, Mizuno Y, et al. Human adhalin is alternatively spliced and the gene is located on chromosome 17q21. Proc Natl Acad Sci USA 1994;91:9690-4.

11 Nigro V, Piluso G, Belsito A, et al. Identification of a novel sarcoglycan gene at $5 \mathrm{q} 33$ encoding a sarcolemmal $35 \mathrm{kT}$ glycoprotein. Hum Mol Genet 1996;5:1179-86.
12 Nigro V, Moreira ES, Piluso G, et al. The 5q autosomal recessive limb-girdle muscular dystrophy (LGMD2F) is caused by a mutation in the $\delta$-sarcoglycan gene. Nat Gene 1996;14:195-8.

13 Campbell K. Three muscular dystrophies: loss of cytoskeleton-extracellular matrix linkage. Cell 1995;80: 675-9.

14 Ozawa E, Yoshida M, Suzuki A, et al. Dystrophin-associated proteins in muscular dystrophy. Hum Mol Genet 1995;4: 1711-16.

15 Worton R. Muscular dystrophies: diseases of the dystrophin-glycoprotein complex. Science 1995;270:755-6.

16 Piccolo F, Roberds SL, Jeanpierre M, et al. Primary adhalinopathy: a common cause of autosomal recessive muscular dystrophy of variable severity. Nat Genet 1995;10 243-5.

17 Carrié A, Piccolo F, de Toma C, et al. Muational diversity and hot spots in the $\alpha$-sarcoglycan gene in autosomal recessive muscular dystrophy (LGMD2D). 7 Med Genet 1997;34:470-5.

18 Passos-Bueno MR, Moreira ES, Vainzof $M$, et al. A common missense mutation in the adhalin gene in three unrelated Brazilian families with a relatively mild form of autosomal recessive limb-girdle muscular dystrophy. Hum Mol Genet 1995;4:1163-7.

19 Bönnemann CG, Passos-Bueno MR, McNally EM, et al. Genomic screening for $\beta$-sarcoglycan gene mutations may cause severe limb-girdle muscular dystrophy type $2 \mathrm{E}$ cause severe limb-girdle muscular dystroph
(LGMD2E). Hum Mol Genet 1996;5:1953-61.

20 McNally EM, Passos-Bueno MR, Bönnemann CG, et al. Mild and severe muscular dystrophy caused by a single $\gamma$-sarcoglycan mutation. Am f Hum Genet 1996;59:1040-7.

21 McNally EM, Duggan D, Gorospe JR, et al. Mutations that disrupt the carboxyl-terminus of $\gamma$-sarcoglycan cause muscular dystrophy. Hum Mol Genet 1996;5:1841-7.

22 Piccolo $F$, Jeanpierre $M$, Leturcq $F$, et al. A founder mutation in the $\gamma$-sarcoglycan gene of Gypsies possibly predating their migration out of India. Hum Mol Genet 1996;12:2019-22.

23 Othmane KB, Speer MC, Stauffer J, et al. Evidence for linkage disequilibrium in chromosome 13-linked Duchennelike muscular dystrophy (LGMD2C). Am f Hum Genet 1995;57:732-4.

24 Duggan DJ, Manchester D, Stears KP, et al. Mutations in the $\delta$-sarcoglycan gene are a rare cause of autosomal recessive limb-girdle muscular dystrophy (LGMD2). Neurogenetics 1997;1:49-58.

25 Vainzof $\mathbf{M}$, Passos-Bueno $\mathbf{M R}$, Canovas $\mathbf{M}$, et al. The sarcoglycan complex in the six autosomal recessive limb-girdle muscular dystrophies. Hum Mol Genet 1996;5: 1963-9

26 Vainzof M, Moreira ES, Ferraz G, Passos-Bueno MR, Marie SK, Zatz M. Further evidence for the organization of the four sarcoglycan proteins within the dystrophinglycoprotein complex (DGC). (Submitted.)

27 Vainzof M, Passos-Bueno MR, Pavanello RCM, Marie SK Zatz M. Sarcoglycanopathies are responsible for $68 \%$ of severe autosomal recessive limb-girdle muscular dystrophy. (Submitted.)

28 Passos-Bueno MR, Vainzof M, Moreira ES, Zatz M. The seven autosomal recessive limb-girdle muscular dystrophies (LGMD): from LGMD2A to LGMD2G. (Submitted.) 